\title{
Blind recognition of space-time block code in MISO system
}

\author{
Guobing Qian ${ }^{1,2 *}$, Liping Li ${ }^{1}$, Minggang Luo ${ }^{1}$, Hongshu Liao ${ }^{1,2}$ and Huaguo Zhang ${ }^{1}$
}

\begin{abstract}
Blind recognition of space-time block codes (STBCs) used in multiple transmitter communication is an important research topic in the non-cooperative scenario, which has attracted more and more attention recently. However, all of the current recognition algorithms can only work well in multiple-input multiple-output system, i.e., the system employs multiple receive antennas. To our knowledge, there is no report in the literature on blind recognition of STBCs in multiple-input single-output (MISO) system, i.e., the system employs only one receive antenna. In this paper, this matter is addressed. An original method of feature extraction for STBCs in the MISO system is proposed using the second-order and higher-order statistics of the reconstructed receiver. After feature extraction, the classification of space-time code can be considered as a pattern recognition problem. A classifier based on a support vector machine is proposed for the recognition of STBCs by mapping these features into a high dimensional space. Simulations show that the proposed classifier can recognize STBCs with high performance and be robust to modulation.
\end{abstract}

Keywords: Blind recognition; Space-time block codes; Multiple-input single-output; SVM

\section{Introduction}

Over the past decade, space-time block codes (STBCs) have been firmly established as an effective technique for achieving reliable transmission in multiple transmitter wireless communication systems [1-3]. The blind recognition of communication parameters of these systems is an important research topic in the non-cooperative scenario, which has attracted more and more attention recently. Most researches are focused on blind channel estimation [4-8]. These blind channel estimation algorithms are based on the assumption that the space-time code is known at the receiver side. However, this is not practical since the space-time code is not known at the receiver side in the non-cooperative scenario. Therefore, one essential step in the signal interception process is to blindly recognize the STBCs used in multiple transmitter communications.

The blind recognition of STBCs is a challenging topic, which is a key research issue in non-cooperative communication systems with military and commercial

*Correspondence: shuaiguobing@hotmail.com

1 School of Electronic Engineering, University of Electronics Science and Technology of China, Chengdu, Sichuan 611731, China

2 Science and Technology on Electronic Information Control Laboratory, Chengdu 610036, China applications. Recently, it has been presented in [9-13] These methods can be divided into three categories: feature-based methods [9-11,13], maximum likelihood (ML) approach [12], and methods derived from ML [12]. These methods can only work well in multiple-input multiple-output (MIMO) system. However, STBCs are generally designed for transmit diversity in the downlink. One of the most appealing researches is to recognize the space-time block codes with a single receive antenna.

To our knowledge, none of the previous algorithms is able to blindly recognize the STBCs with a single receive antenna. In this paper, an original support vector machine (SVM)-based classifier is proposed which is well suited for this case. The received one-dimensional signal is reconstructed as a multidimensional signal, and then the SVM-based classifier is proposed based on second-order and higher-order statistics of the reconstructed receiver. The method proposed in this paper can be easily implemented without a priori knowledge about the number of transmitters, the modulation of the transmitted symbols, and the channel state information (CSI). The only assumption lies on the perfect estimation of timing synchronization (one sample per symbol, optimum sampling time). The candidate STBCs for recognition, which are the

\section{Springer}

C 2013 Qian et al - licensee Springer. This is an Open Access article distributed under the terms of the Creative Commons

Attribution License (http://creativecommons.org/licenses/by/2.0), which permits unrestricted use, distribution, and reproduction in any medium, provided the original work is properly cited. 
same as those in [10], include spatial multiplexing (SM), Alamouti code, orthogonal STBC using three transmit antennas (OSTBC3)with rate 3/4 (first and second codes), and OSTBC3 with rate $1 / 2$. These codes belong to the class of linear space-time block codes, and transmitted matrices of these codes are presented in Section 2.

The remainder of this paper is organized as follows. The system model of communication is introduced in Section 2. Feature extractions based on the second-order and higher-order statistics of the reconstructed receiver of different STBCs are presented in Section 3. It is shown that the second-order or higherorder statistics of the reconstructed receiver presents distinguished features. Based on these features, Section 4 proposes an SVM-based classifier for the blind recognition of five linear STBCs. Finally, simulation results are presented in Section 5, and conclusions are drawn in Section 6.

Notations adopted in this paper are mostly standard. Uppercase and bold letters denote matrices, lower case and bold letters denote vectors, and lower case and nonbold letters denote scalars. Superscript $(\cdot)^{T},(\cdot)^{*},(\cdot)^{H}$, and $(\cdot)^{\dagger}$ stand for transpose, complex conjugate, Hermitian transpose, and pseudo-inverse, respectively. The operator $\operatorname{Re}(\cdot)$ denotes the real parts. The operator $E[\cdot]$ denotes the expectation, and $\|\cdot\|_{F}$ represents the Frobenius norm.

\section{System models}

\subsection{Signal models of linear space-time block codes}

In a linear STBC communications system of multiple transmitters, a signal vector $\mathbf{s}_{v}=\left[s_{1}, \cdots, s_{n}\right]^{T}(v=$ $\left.1, \cdots, N_{b}\right)$ composed of $n$ symbols is encoded into a $n_{t} \times l$ transmitted matrix $\mathbf{C}\left(\mathbf{s}_{v}\right)$, where $N_{b}$ corresponds to the number of transmitted blocks, $n_{t}$ and $l$ denote the number of transmitting antennas and the length of a block, respectively. Furthermore, the transmitted symbols $\mathbf{s}$ are assumed to be zero mean, non-Gaussian, independent, and identically distributed (i.i.d.), and they belong to the same linear modulation scheme (pluggable authentication module (PAM), quadrature amplitude modulation (QAM), or phase-shift keying (PSK)). Parameters $n, n_{t}$, and $l$ depend only on the STBC employed at the transmitter side.

The candidate STBCs for recognition include SM, Alamouti code, OSTBC3 with rate $3 / 4$ (first and second codes), and OSTBC3 with rate $1 / 2$. The transmitted matrices of these STBCs are as follows:

1. Spatial multiplexing

$$
\mathbf{C}\left(\mathbf{s}_{v}\right)=\left[s_{1} \cdots s_{n_{t}}\right]^{T}
$$

2. Alamouti

$$
\mathbf{C}\left(\mathbf{s}_{v}\right)=\left[\begin{array}{cc}
s_{1} & -s_{2}^{*} \\
s_{2} & s_{1}^{*}
\end{array}\right]
$$

3. First code of OSTBC3 with rate $3 / 4$

$$
\mathbf{C}\left(\mathbf{s}_{v}\right)=\left[\begin{array}{cccc}
s_{1} & 0 & s_{2} & -s_{3} \\
0 & s_{1} & s_{3}^{*} & s_{2}^{*} \\
-s_{2}^{*} & -s_{3} & s_{1} & 0
\end{array}\right]
$$

4. Second code of OSTBC3 with rate $3 / 4$

$$
\mathbf{C}\left(\mathbf{s}_{v}\right)=\left[\begin{array}{cccc}
s_{1} & -s_{2}^{*} & s_{3}^{*} & 0 \\
s_{2} & s_{1}^{*} & 0 & -s_{3}^{*} \\
s_{3} & 0 & -s_{1}^{*} & s_{2}^{*}
\end{array}\right]
$$

5. OSTBC3 with rate $1 / 2$

$$
\mathbf{C}\left(\mathbf{s}_{v}\right)=\left[\begin{array}{cccccccc}
s_{1} & -s_{2} & -s_{3} & -s_{4} & s_{1}^{*} & -s_{2}^{*} & -s_{3}^{*} & -s_{4}^{*} \\
s_{2} & s_{1} & s_{4} & -s_{3} & s_{2}^{*} & s_{1}^{*} & s_{4}^{*} & -s_{3}^{*} \\
s_{3} & -s_{4} & s_{1} & s_{2} & s_{3}^{*} & -s_{4}^{*} & s_{1}^{*} & s_{2}^{*}
\end{array}\right]
$$

\subsection{System models of the multiple-input single-output communication}

In this paper, we assume that transmitted signals are narrow band, and the channel is quasi-static and frequency flat. Moreover, the receiver is perfectly synchronized with the transmitter, i.e., one sample per symbol and an optimum sampling time. In this case, the multiple-input single-output (MISO) communication model of the $v$ th block is expressed as follows [1]:

$$
\mathbf{y}_{v}=\mathbf{h C}\left(\mathbf{s}_{\mathbf{v}}\right)+\mathbf{w}_{v}
$$

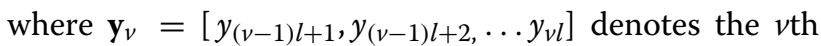
block of received samples, $\mathbf{h}=\left[h_{1}, h_{2} \ldots h_{n_{t}}\right]$ represents the channel between the transmit antennas and receive antenna, and $\mathbf{w}_{v}=\left[w_{(v-1) l+1}, w_{(v-1) l+2}, \ldots w_{v l}\right]$ refers to the $v$ th block of additive receiver noise, which is assumed to be white and Gaussian.

Our aim is to blindly recognize the space-time block code of a communication with a single receive antenna from $N$ received samples. Hereafter in this paper, $N$ is assumed to be an integer multiple of 4 to allow simplifications of the following mathematical expressions. Moreover, if this assumption does not hold, the excess samples can be discarded to meet this assumption easily. 


\section{Feature extraction}

In this section, we reconstruct the receiver and analyze the second- or higher-order statistics of the reconstructed receiver. Feature extractions of different STBCs are based on the second- and higher-order statistics of the reconstructed receiver.

\subsection{Feature extraction of SM's coded signal}

Based on the assumptions of Section 2, it can be easily obtained that received samples of SM's coded signal can be expressed as follows:

$$
\begin{aligned}
\mathbf{y}= & {\left[\begin{array}{llll}
y_{1} & y_{2} & \cdots & y_{N}
\end{array}\right]=\mathbf{h C}(\mathbf{s})+\mathbf{w} } \\
= & {\left[\begin{array}{llll}
h_{1} & h_{2} & \cdots & h_{n_{t}}
\end{array}\right]\left[\begin{array}{cccc}
s_{1} & s_{n_{t}+1} & \cdots & s_{n_{t}(N-1)+1} \\
s_{2} & s_{n_{t}+2} & \cdots & s_{n_{t}(N-1)+2} \\
\vdots & \vdots & \vdots & \vdots \\
s_{n_{t}} & s_{2 n_{t}} & \cdots & s_{n_{t}} N
\end{array}\right] } \\
& +\left[\begin{array}{llll}
w_{1} & w_{2} & \cdots & w_{N}
\end{array}\right] .
\end{aligned}
$$

1. If the received samples are reshaped as follows:

$$
\mathbf{Y}_{1}=\left[\begin{array}{llll}
y_{1} & y_{5} & \cdots & y_{N-3} \\
y_{2} & y_{6} & \cdots & y_{N-2} \\
y_{3} & y_{7} & \cdots & y_{N-1} \\
y_{4} & y_{8} & \cdots & y_{N}
\end{array}\right]
$$

then the reshaped samples can be expressed as:

$$
\begin{aligned}
& \mathbf{Y}_{1}=\left[\begin{array}{cccccccccccc}
h_{1} & \cdots & h_{n_{t}} & 0 & \cdots & 0 & 0 & \cdots & 0 & 0 & \cdots & 0 \\
0 & \cdots & 0 & h_{1} & \cdots & h_{n_{t}} & 0 & \cdots & 0 & 0 & \cdots & 0 \\
0 & \cdots & 0 & 0 & \cdots & 0 & h_{1} & \cdots & h_{n_{t}} & 0 & \cdots & 0 \\
0 & \cdots & 0 & 0 & \cdots & 0 & 0 & \cdots & 0 & h_{1} & \cdots & h_{n_{t}}
\end{array}\right] \\
& \times\left[\begin{array}{ccc}
s_{1} & \cdots & s_{\left(n_{t}-4\right) N+1} \\
\vdots & \vdots & \vdots \\
s_{n_{t}} & \cdots & s_{\left(n_{t}-3\right) N} \\
s_{n_{t}+1} & \cdots & s_{\left(n_{t}-3\right) N+1} \\
\vdots & \vdots & \vdots \\
s_{2 n_{t}} & \cdots & s_{\left(n_{t}-2\right) N} \\
s_{2 n_{t}+1} & \cdots & s_{\left(n_{t}-2\right) N+1} \\
\vdots & \vdots & \vdots \\
s_{3 n_{t}} & \cdots & s_{\left(n_{t}-1\right) N} \\
s_{3 n_{t}+1} & \cdots & s_{\left(n_{t}-1\right) N+1} \\
\vdots & \vdots & \vdots \\
s_{4 n_{t}} & \cdots & s_{n_{t}} N
\end{array}\right] \\
& +\left[\begin{array}{llll}
w_{1} & w_{5} & \cdots & w_{N-3} \\
w_{2} & w_{6} & \cdots & w_{N-2} \\
w_{3} & w_{7} & \cdots & w_{N-1} \\
w_{4} & w_{8} & \cdots & w_{N}
\end{array}\right]
\end{aligned}
$$

Based on the independence of $s_{i}$, we can easily get that

$$
\begin{aligned}
\mathbf{R}_{1} & =\lim _{N \rightarrow \infty}\left[\frac{1}{N / 4} \mathbf{Y}_{1} \mathbf{Y}_{1}^{H}\right] \\
& =\left[\left(\left|h_{1}\right|^{2}+\cdots\left|h_{n_{t}}\right|^{2}\right) E\left(|s|^{2}\right)+\sigma^{2}\right] \mathrm{I}_{4} .
\end{aligned}
$$

In practice, $\mathbf{R}_{1}$ can only be estimated by limited received samples $N$. Therefore, we define performance index $I_{1}\left(\mathbf{R}_{1}\right)=$

$\frac{1}{2 M(M-1)}\left[\sum_{i=1}^{M}\left(\sum_{j=1}^{M} \frac{\left|r_{i j}\right|}{\left|r_{i i}\right|}-1\right)+\sum_{j=1}^{M}\left(\sum_{i=1}^{M} \frac{\left|r_{i j}\right|}{\left|r_{j j}\right|}-1\right)\right]$ to measure the feature of $\mathbf{R}_{1}$, where $M=4$ denotes the order of matrix $\mathbf{R}_{1}, r_{i, j}$ denotes the element on the $i$ th row and the $j$ th column of matrix $\mathbf{R}_{1}$. The lower the $I_{1}\left(\mathbf{R}_{1}\right)$ value, the better of the performance. This performance index is similar to the Amari index defined in [14].

2. If the received samples are reconstructed as follows:

$$
\mathbf{Y}_{2}=\left[\begin{array}{cccc}
y_{1} & y_{3} & \cdots & y_{N-1} \\
y_{2}^{*} & y_{4}^{*} & \cdots & y_{N}^{*}
\end{array}\right]
$$

then the reconstructed samples can be expressed as:

$$
\begin{aligned}
& \mathbf{Y}_{2}= \underbrace{\left[\begin{array}{cccccc}
h_{1} & \cdots & h_{n_{t}} & 0 & \cdots & 0 \\
0 & \cdots & 0 & h_{1}^{*} & \cdots & h_{n_{t}}^{*}
\end{array}\right]}_{\mathbf{H}} \underbrace{\left[\begin{array}{cccc}
s_{1} & \cdots & s_{\left(n_{t}-2\right) N+1} \\
\vdots & \vdots & \vdots \\
s_{n_{t}} & \cdots & s_{\left(n_{t}-1\right) N} \\
s_{n_{t}+1}^{*} & \cdots & s_{\left(n_{t}-1\right) N+1}^{*} \\
\vdots & \vdots & \vdots \\
s_{2 n_{t}}^{*} & \cdots & s_{n_{t} N}^{*}
\end{array}\right]}_{\mathbf{S}} \\
&+\left[\begin{array}{llll}
w_{1} & w_{3} & \cdots & w_{N-1} \\
w_{2}^{*} & w_{4}^{*} & \cdots & w_{N}^{*}
\end{array}\right] .
\end{aligned}
$$

It can be easily obtained that

$$
\begin{aligned}
& \mathbf{C}_{4}^{[1,2]}=c_{4}\left[\mathbf{Y}_{2}(:, i), \quad \mathbf{Y}_{2}(:, i)^{H}, \quad \mathbf{Y}_{2}(1, i), \quad \mathbf{Y}_{2}(2, i)^{H}\right] \\
& =\left[\begin{array}{llll}
c_{4}\left[\mathbf{Y}_{2}(1, i),\right. & \mathbf{Y}_{2}(1, i)^{H}, & \mathbf{Y}_{2}(1, i), & \mathbf{Y}_{2}(2, i)^{H} \\
c_{4}\left[\mathbf{Y}_{2}(2, i),\right. & \mathbf{Y}_{2}(1, i)^{H}, & \mathbf{Y}_{2}(1, i), & \mathbf{Y}_{2}(2, i)^{H}
\end{array}\right] \\
& \left.\begin{array}{llll}
c_{4}\left[\mathbf{Y}_{2}(1, i),\right. & \mathbf{Y}_{2}(2, i)^{H}, & \mathbf{Y}_{2}(1, i), & \left.\mathbf{Y}_{2}(2, i)^{H}\right] \\
c_{4}\left[\mathbf{Y}_{2}(2, i),\right. & \mathbf{Y}_{2}(2, i)^{H}, & \mathbf{Y}_{2}(1, i), & \mathbf{Y}_{2}(2, i)^{H}
\end{array}\right] \\
& =\left(\begin{array}{ll}
0 & 0 \\
0 & 0
\end{array}\right) \text {, }
\end{aligned}
$$


where $\mathbf{Y}_{2}(i, j)$ denotes the element on the $i$ th row and the $j$ th column of $\mathbf{Y}_{2}, \mathbf{Y}_{2}(:, i)$ denotes the elements on the ith column of $\mathbf{Y}_{2}, c_{4}\left(x_{1}, x_{2}, x_{3}, x_{4}\right)$ is defined as $c_{4}\left(x_{1}, x_{2}, x_{3}, x_{4}\right)=E\left(x_{1} x_{2}^{*} x_{3} x_{4}^{*}\right)-$ $E\left(x_{1} x_{2}^{*}\right) E\left(x_{3} x_{4}^{*}\right)-E\left(x_{1} x_{3}^{*}\right) E\left(x_{2} x_{4}^{*}\right)-E\left(x_{1} x_{4}^{*}\right) E\left(x_{2} x_{3}^{*}\right)$. For proof of (13), see Appendix 1.

\subsection{Feature extraction of an Alamouti's coded signal}

The received samples of Alamouti's coded signal can be expressed as follows:

$$
\begin{aligned}
& \mathbf{y}=\left[\begin{array}{llll}
\mathbf{y}_{1} & \mathbf{y}_{2} & \cdots & \mathbf{y}_{N_{b}}
\end{array}\right]=\left[\begin{array}{llll}
y_{1} & y_{2} & \cdots & y_{N}
\end{array}\right] \\
& =\mathbf{h C}(\mathbf{s})+\mathbf{w}=\left[h_{1} h_{2}\right]\left[\begin{array}{ccccccc}
s_{1} & -s_{2}^{*} & s_{3} & -s_{4}^{*} & \cdots & s_{N-1} & -s_{N}^{*} \\
s_{2} & s_{1}^{*} & s_{4} & s_{3}^{*} & \cdots & s_{N} & s_{N-1}^{*}
\end{array}\right] \\
& +\left[\begin{array}{llll}
w_{1} & w_{2} & \cdots & w_{N}
\end{array}\right] .
\end{aligned}
$$

1. If the received samples are reshaped as (8), then the reshaped samples can be expressed as follows:

$$
\begin{aligned}
\mathbf{Y}_{1}= & {\left[\begin{array}{cccccccc}
h_{1} & h_{2} & 0 & 0 & 0 & 0 & 0 & 0 \\
0 & 0 & h_{1} & h_{2} & 0 & 0 & 0 & 0 \\
0 & 0 & 0 & 0 & h_{1} & h_{2} & 0 & 0 \\
0 & 0 & 0 & 0 & 0 & 0 & h_{1} & h_{2}
\end{array}\right]\left[\begin{array}{ccc}
s_{1} & \cdots & s_{N-3} \\
s_{2} & \cdots & s_{N-2} \\
-s_{2}^{*} & \cdots & -s_{N-2}^{*} \\
s_{1}^{*} & \cdots & s_{N-3}^{*} \\
s_{3} & \cdots & s_{N-1} \\
s_{4} & \cdots & s_{N} \\
-s_{4}^{*} & \cdots & -s_{N}^{*} \\
s_{3}^{*} & \cdots & s_{N-1}^{*}
\end{array}\right] } \\
& +\left[\begin{array}{llll}
w_{1} & w_{5} & \cdots & w_{N-3} \\
w_{2} & w_{6} & \cdots & w_{N-2} \\
w_{3} & w_{7} & \cdots & w_{N-1} \\
w_{4} & w_{8} & \cdots & w_{N}
\end{array}\right] .
\end{aligned}
$$

(i) If $s_{i}$ belongs to modulation scheme I ( $\geq 4$ PSK or 4 QAM or 16 QAM or 32 QAM), then $E\left(s_{i} s_{i}\right)=E\left(s_{i}^{*} s_{i}^{*}\right)=0$. It can be easily obtained that

$\mathbf{R}_{1}=\lim _{N \rightarrow \infty}\left[\frac{1}{N / 4} \mathbf{Y}_{1} \mathbf{Y}_{1}{ }^{H}\right]=\left[\left(\left|h_{1}\right|^{2}+\left|h_{2}\right|^{2}\right) E\left(|s|^{2}\right)+\sigma^{2}\right] \mathrm{I}_{4}$.

(ii) If $s_{i}$ belongs to modulation scheme II (BPSK or 8QAM or PAM), then $E\left(s_{i} s_{i}\right) \neq 0, E\left(s_{i}^{*} s_{i}^{*}\right) \neq 0$. It can be easily obtained that

$$
\begin{aligned}
\mathbf{R}_{1} & =\lim _{N \rightarrow \infty}\left[\frac{1}{N / 4} \mathbf{Y}_{1} \mathbf{Y}_{1}{ }^{H}\right] \\
& =\left[\begin{array}{cccc}
\left(\left|h_{1}\right|^{2}+\left|h_{2}\right|^{2}\right) E\left(|s|^{2}\right)+\sigma^{2} & \left(h_{1} h_{2}^{*}-h_{1}^{*} h_{2}\right) E\left(s^{2}\right) & 0 & 0 \\
\left(h_{1}^{*} h_{2}-h_{1} h_{2}^{*}\right) E\left(s^{* 2}\right) & \left(\left|h_{1}\right|^{2}+\left|h_{2}\right|^{2}\right) E\left(|s|^{2}\right)+\sigma^{2} & 0 & 0 \\
0 & 0 & \left(\left|h_{1}\right|^{2}+\left|h_{2}\right|^{2}\right) E\left(|s|^{2}\right)+\sigma^{2} & \left(h_{1} h_{2}^{*}-h_{1}^{*} h_{2}\right) E\left(s^{2}\right) \\
0 & 0 & \left(h_{1}^{*} h_{2}-h_{1} h_{2}^{*}\right) E\left(s^{* 2}\right) & \left(\left|h_{1}\right|^{2}+\left|h_{2}\right|^{2}\right) E\left(|s|^{2}\right)+\sigma^{2}
\end{array}\right] .
\end{aligned}
$$

In order to measure the feature of $\mathbf{R}_{1}$, we partition it as $\mathbf{R}_{1}=\left[\begin{array}{ll}\mathbf{P}_{1} & \mathbf{P}_{2} \\ \mathbf{P}_{3} & \mathbf{P}_{4}\end{array}\right]$, where $\mathbf{P}_{1}, \mathbf{P}_{2}$, $\mathbf{P}_{3}$, and $\mathbf{P}_{4}$ are $2 \times 2$ dimensional matrix. The feature of $\mathbf{R}_{1}$ can be measured by performance index $I_{2}\left(\mathbf{R}_{1}\right)=$ $\frac{1}{4}\left[\frac{\left\|\mathbf{P}_{3}\right\|_{F}+\left\|\mathbf{P}_{2}\right\|_{F}}{\left\|\mathbf{P}_{1}\right\|_{F}}+\frac{\left\|\mathbf{P}_{3}\right\|_{F}+\left\|\mathbf{P}_{2}\right\|_{F}}{\left\|\mathbf{P}_{4}\right\|_{F}}\right]$. The lower the $I_{2}\left(\mathbf{R}_{1}\right)$ value, the better the performance.

2. If the received samples are reconstructed as (11), then the reconstructed samples can be expressed as follows:

$$
\mathbf{Y}_{2}=\underbrace{\left[\begin{array}{ll}
h_{1} & h_{2} \\
h_{2}^{*} & -h_{1}^{*}
\end{array}\right]}_{\mathbf{H}_{1}}\left[\begin{array}{llll}
s_{1} & s_{3} & \cdots & s_{N-1} \\
s_{2} & s_{4} & \cdots & s_{N}
\end{array}\right]+\left[\begin{array}{cccc}
w_{1} & w_{3} & \cdots & w_{N-1} \\
w_{2}^{*} & w_{4}^{*} & \cdots & w_{N}^{*}
\end{array}\right] \text {. }
$$

It can be easily obtained that

$$
\begin{aligned}
\mathbf{C}_{4}^{[1,2]} & =c_{4}\left[\mathbf{Y}_{2}(:, i), \quad \mathbf{Y}_{2}(:, i)^{H}, \quad \mathbf{Y}_{2}(1, i), \quad \mathbf{Y}_{2}(2, i)^{H}\right] \\
& =k_{4} h_{1} h_{2} \mathbf{H}_{1}\left[\begin{array}{cc}
1 & 0 \\
0 & -1
\end{array}\right] \mathbf{H}_{1}{ }^{H}, \\
& \text { where } \mathrm{k}_{4}=\mathrm{c}_{4}\left(\mathrm{~s}_{1}, \mathrm{~s}_{1}, \mathrm{~s}_{1}, \mathrm{~s}_{1}\right)=\mathrm{c}_{4}\left(\mathrm{~s}_{2}, \mathrm{~s}_{2}, \mathrm{~s}_{2}, \mathrm{~s}_{2}\right)
\end{aligned}
$$

For the proof of (19), see Appendix 2.

Therefore, if $\mathbf{C}_{4}^{[k, j]}$ is decomposed as $\mathbf{C}_{4}^{[k, j]}=\mathbf{U} \Lambda \mathbf{U}^{H}$, then the relationship between $\mathbf{U}$ and $\mathbf{H}_{1}$ can be expressed as $\mathbf{H}_{1}=\mathbf{U} \mathbf{P}_{1} \Lambda_{1}$, where $\mathbf{P}_{1}$ and $\Lambda_{1}$ denote permutation matrix and diagonal matrix, respectively. Moreover, matrix $\mathbf{H}_{1}$ can be identified from $\mathbf{Y}_{2}$ by independent component analysis (ICA)-based method (for example, joint approximate diagonalization of eigenmatrices (JADE) [15]). It should be noted that the relationship between $\mathbf{H}_{1}$ and $\hat{\mathbf{H}}_{1}$ (which denotes the identification of matrix $\mathbf{H}_{1}$ by ICA-based method) can be expressed as $\mathbf{H}_{1}=\hat{\mathbf{H}}_{1} \mathbf{P}_{2} \Lambda_{2}$, where $\mathbf{P}_{2}$ and $\Lambda_{2}$ denote permutation matrix and diagonal matrix, respectively. Therefore, the feature of $\mathbf{T}=\mathbf{U}^{\dagger} \hat{\mathbf{H}}_{1}=\mathbf{P}_{1} \Lambda_{1} \Lambda_{2}^{-1} \mathbf{P}_{2}^{-1}$ can be measured by Amari index $I(\mathbf{T})=\frac{1}{2 M(M-1)}\left[\sum_{i=1}^{M}\left(\sum_{j=1}^{M} \frac{\left|t_{i j}\right|}{\max _{k}\left|t_{i k}\right|}-1\right)\right.$ $\left.+\sum_{j=1}^{M}\left(\sum_{i=1}^{M} \frac{\left|t_{i j}\right|}{\max _{k}\left|t_{k j}\right|}-1\right)\right]$ defined in [14], where $M=4$ denotes the order of matrix $\mathbf{T}, t_{i, j}$ denotes the element on the ith row and the $j$ th column of matrix $\mathbf{T}$. 


\subsection{Feature extraction of code of OSTBC3 with rate 3/4's coded signal}

The received samples of the first code of OSTBC3 with rate 3/4's coded signal can be expressed as:

$$
\mathbf{y}=\left[\begin{array}{lll}
h_{1} & h_{2} & h_{3}
\end{array}\right]\left[\begin{array}{ccccccccc}
s_{1} & 0 & s_{2} & -s_{3} & \cdots & s_{3 N_{b}-2} & 0 & s_{3 N_{b}-1} & -s_{3 N_{b}} \\
0 & s_{1} & s_{3}^{*} & s_{2}^{*} & \cdots & 0 & s_{3 N_{b}-2} & s_{3 N_{b}}^{*} & s_{3 N_{b}-1}^{*} \\
-s_{2}^{*} & -s_{3} & s_{1} & 0 & \cdots & -s_{3 N_{b}-1}^{*} & -s_{N_{b}} & s_{3 N_{b}-2} & 0
\end{array}\right]+\left[\begin{array}{llll}
w_{1} & w_{2} & \cdots & w_{N}
\end{array}\right] .
$$

Here, $N_{b}=\frac{N}{4}$ denotes the number of transmission blocks.

1. If the received samples are reshaped as (8), then the reshaped samples can be expressed as:

$$
\mathbf{Y}_{1}=\left[\begin{array}{cccccc}
h_{1} & 0 & 0 & 0 & -h_{3} & 0 \\
h_{2} & 0 & -h_{3} & 0 & 0 & 0 \\
h_{3} & h_{1} & 0 & 0 & 0 & h_{2} \\
0 & 0 & -h_{1} & 0 & h_{2} & 0
\end{array}\right]\left[\begin{array}{cccc}
s_{1} & s_{4} & \cdots & s_{3 N_{b}-2} \\
s_{2} & s_{5} & \cdots & s_{3 N_{b}-1} \\
s_{3} & s_{6} & \cdots & s_{3 N_{b}} \\
s_{1}^{*} & s_{4}^{*} & \cdots & s_{3 N_{b}-2}^{*} \\
s_{2}^{*} & s_{5}^{*} & \cdots & s_{3 N_{b}-1}^{*} \\
s_{3}^{*} & s_{6}^{*} & \cdots & s_{3 N_{b}}^{*}
\end{array}\right]+\left[\begin{array}{cccc}
w_{1} & w_{5} & \cdots & w_{N-3} \\
w_{2} & w_{6} & \cdots & w_{N-2} \\
w_{3} & w_{7} & \cdots & w_{N-1} \\
w_{4} & w_{8} & \cdots & w_{N}
\end{array}\right] .
$$

(i) If $s_{i}$ belongs to modulation scheme I, then it can be easily obtained that

$$
\begin{aligned}
\mathbf{R}_{1} & =\lim _{N \rightarrow \infty}\left[\frac{1}{N / 4} \mathbf{Y}_{1} \mathbf{Y}_{1}^{H}\right] \\
& =E\left(|s|^{2}\right)\left[\begin{array}{cccc}
\left|h_{1}\right|^{2}+\left|h_{3}\right|^{2} & h_{1} h_{2}^{*} & h_{1} h_{3}^{*} & -h_{2}^{*} h_{3} \\
h_{1}^{*} h_{2} & \left|h_{2}\right|^{2}+\left|h_{3}\right|^{2} & h_{2} h_{3}^{*} & h_{1}^{*} h_{3} \\
h_{1}^{*} h_{3} & h_{2}^{*} h_{3} & \left|h_{1}\right|^{2}+\left|h_{2}\right|^{2}+\left|h_{3}\right|^{2} & 0 \\
-h_{2} h_{3}^{*} & h_{1} h_{3}^{*} & 0 & \left|h_{1}\right|^{2}+\left|h_{2}\right|^{2}
\end{array}\right]+\sigma^{2} \mathbf{I}_{4} .
\end{aligned}
$$

(ii) If $s_{i}$ belongs to modulation scheme II, then it can be easily obtained that

$$
\begin{aligned}
\mathbf{R}_{1}= & \lim _{N \rightarrow \infty}\left[\frac{1}{N / 4} \mathbf{Y}_{1} \mathbf{Y}_{1}{ }^{H}\right] \\
= & {\left[\begin{array}{cccc}
\left(\left|h_{1}\right|^{2}+\left|h_{3}\right|^{2}\right) E\left(|s|^{2}\right)+\sigma^{2} & h_{1} h_{2}^{*} E\left(|s|^{2}\right) & h_{1} h_{3}^{*} E\left(|s|^{2}\right)-h_{1}^{*} h_{3} E\left(s^{* 2}\right) & -h_{2}^{*} h_{3} E\left(|s|^{2}\right) \\
h_{1}^{*} h_{2} E\left(|s|^{2}\right) & \left(\left|h_{2}\right|^{2}+\left|h_{3}\right|^{2}\right) E\left(|s|^{2}\right)+\sigma^{2} & h_{2} h_{3}^{*} E\left(|s|^{2}\right)-h_{2}^{*} h_{3} E\left(s^{2}\right) & h_{1}^{*} h_{3} E\left(|s|^{2}\right) \\
h_{1}^{*} h_{3} E\left(|s|^{2}\right)-h_{1} h_{3}^{*} E\left(s^{2}\right) & -h_{2} h_{3}^{*} E\left(s^{* 2}\right)+h_{2}^{*} h_{3} E\left(|s|^{2}\right) & \left(\left|h_{1}\right|^{2}+\left|h_{2}\right|^{2}+\left|h_{3}\right|^{2}\right) E\left(|s|^{2}\right)+\sigma^{2} & h_{1} h_{2}^{*} E\left(s^{2}\right)-h_{1}^{*} h_{2} E\left(s^{* 2}\right) \\
-h_{2} h_{3}^{*} E\left(|s|^{2}\right) & h_{1} h_{3}^{*} E\left(|s|^{2}\right) & h_{1}^{*} h_{2} E\left(s^{* 2}\right)-h_{1} h_{2}^{*} E\left(s^{2}\right) & \left(\left|h_{1}\right|^{2}+\left|h_{2}\right|^{2}\right) E\left(|s|^{2}\right)+\sigma^{2}
\end{array}\right] . }
\end{aligned}
$$

2. If the received samples are reconstructed as follows:

$$
\mathbf{Y}_{3}=\left[\begin{array}{llll}
y_{1} & y_{5} & \cdots & y_{N-3} \\
y_{2} & y_{6} & \cdots & y_{N-2} \\
y_{3}^{*} & y_{7}^{*} & \cdots & y_{N-1}^{*} \\
y_{4} & y_{8} & \cdots & y_{N}
\end{array}\right]
$$

then the reconstructed samples can be expressed as:

$$
\mathbf{Y}_{3}=\left[\begin{array}{ccc}
h_{1} & -h_{3} & 0 \\
h_{2} & 0 & -h_{3} \\
h_{3}^{*} & h_{1}^{*} & h_{2}^{*} \\
0 & h_{2} & -h_{1}
\end{array}\right]\left[\begin{array}{cccc}
s_{1} & s_{4} & \cdots & s_{3 N_{b}-2} \\
s_{2}^{*} & s_{5}^{*} & \cdots & s_{3 N_{b}-1}^{*} \\
s_{3} & s_{6} & \cdots & s_{3 N_{b}}
\end{array}\right]+\left[\begin{array}{cccc}
w_{1} & w_{5} & \cdots & w_{N-3} \\
w_{2} & w_{6} & \cdots & w_{N-2} \\
w_{3}^{*} & w_{7}^{*} & \cdots & w_{N-1}^{*} \\
w_{4} & w_{8} & \cdots & w_{N}
\end{array}\right] .
$$

It can be easily obtained that

$$
\begin{aligned}
\mathbf{R}_{3} & =\lim _{N \rightarrow \infty}\left[\frac{1}{N / 4} \mathbf{Y}_{3} \mathbf{Y}_{3}^{H}\right] \\
& =E\left(|s|^{2}\right)\left[\begin{array}{cccc}
\left(\left|h_{1}\right|^{2}+\left|h_{3}\right|^{2}\right) & h_{1} h_{2}^{*} & 0 & -h_{2}^{*} h_{3} \\
h_{1}^{*} h_{2} & \left(\left|h_{2}\right|^{2}+\left|h_{3}\right|^{2}\right) & 0 & h_{1}^{*} h_{3} \\
0 & 0 & \left(\left|h_{1}\right|^{2}+\left|h_{2}\right|^{2}+\left|h_{3}\right|^{2}\right) & 0 \\
-h_{2} h_{3}^{*} & h_{1} h_{3}^{*} & 0 & \left(\left|h_{1}\right|^{2}+\left|h_{2}\right|^{2}\right)
\end{array}\right]+\sigma^{2} \mathbf{I}_{4}
\end{aligned}
$$


The feature of $\mathbf{R}_{3}$ can be measured by $I_{3}\left(\mathbf{R}_{3}\right)=\frac{1}{2(M-1)}\left[\left(\sum_{j=1}^{M} \frac{\left|r_{3,3}\right|}{\left|r_{3,3}\right|}-1\right)+\left(\sum_{i=1}^{M} \frac{\left|r_{i, 3}\right|}{\left|r_{3,3}\right|}-1\right)\right]$, where $M=4$ denotes the order of matrix $\mathbf{R}_{3}, r_{i, j}$ denotes the element on the $i$ th row and the $j$ th column of matrix $\mathbf{R}_{3}$. The lower the $I_{3}(\mathbf{R} 3)$ value, the better the performance.

\subsection{Feature extraction of the second code of OSTBC 3 with rate $3 / 4$ 's coded signal}

The received samples of the second code of OSTBC3 with rate 3/4's coded signal can be expressed as:

$$
\begin{aligned}
& \mathbf{y}=\left[\begin{array}{llll}
y_{1} & y_{2} & \cdots & y_{N}
\end{array}\right]=\mathbf{h C}(\mathbf{s})+\mathbf{w} \\
& =\left[\begin{array}{lll}
h_{1} & h_{2} & h_{3}
\end{array}\right]\left[\begin{array}{ccccccccc}
s_{1} & -s_{2}^{*} & s_{3}^{*} & 0 & \cdots & s_{3 N_{b}-2} & -s_{3 N_{b}-1}^{*} & s_{3 N_{b}}^{*} & 0 \\
s_{2} & s_{1}^{*} & 0 & -s_{3}^{*} & \cdots & s_{3 N_{b}-1} & s_{3 N_{b}-2} & 0 & -s_{3 N_{b}}^{*} \\
s_{3} & 0 & -s_{1}^{*} & s_{2}^{*} & \cdots & s_{3 N_{b}} & 0 & -s_{3 N_{b}-2}^{*} & s_{3 N_{b}-1}^{*}
\end{array}\right]+\left[\begin{array}{lllll}
w_{1} & w_{2} & \cdots & w_{N}
\end{array}\right]
\end{aligned}
$$

Here, $N_{b}=\frac{N}{4}$ denotes the number of transmission blocks.

1. If the received samples are reshaped as (8), then the reshaped samples can be expressed as:

$$
\mathbf{Y}_{1}=\left[\begin{array}{cccccc}
h_{1} & h_{2} & h_{3} & 0 & 0 & 0 \\
0 & 0 & 0 & h_{2} & -h_{1} & 0 \\
0 & 0 & 0 & -h_{3} & h_{1} & 0 \\
0 & 0 & 0 & h_{3} & -h_{2} & 0
\end{array}\right]\left[\begin{array}{cccc}
s_{1} & s_{4} & \cdots & s_{3 N_{b}-2} \\
s_{2} & s_{5} & \cdots & s_{3 N_{b}-1} \\
s_{3} & s_{6} & \cdots & s_{3 N_{b}} \\
s_{1}^{*} & s_{4}^{*} & \cdots & s_{3 N_{b}-2}^{*} \\
s_{2}^{*} & s_{5}^{*} & \cdots & s_{3 N_{b}-1}^{*} \\
s_{3}^{*} & s_{6}^{*} & \cdots & s_{3 N_{b}}^{*}
\end{array}\right]+\left[\begin{array}{cccc}
w_{1} & w_{5} & \cdots & w_{N-3} \\
w_{2} & w_{6} & \cdots & w_{N-2} \\
w_{3} & w_{7} & \cdots & w_{N-1} \\
w_{4} & w_{8} & \cdots & w_{N}
\end{array}\right]
$$

(i) If $s_{i}$ belongs to modulation scheme I, then it can be easily obtained that

$$
\mathbf{R}_{1}=\lim _{N \rightarrow \infty}\left[\frac{1}{N / 4} \mathbf{Y}_{1} \mathbf{Y}_{1}^{H}\right]=\left[E\left(|s|^{2}\right)\right]\left[\begin{array}{cccc}
\left|h_{1}\right|^{2}+\left|h_{2}\right|^{2}+\left|h_{3}\right|^{2} & 0 & 0 & 0 \\
0 & \left|h_{1}\right|^{2}+\left|h_{2}\right|^{2} & -h_{2} h_{3}^{*}-\left|h_{1}\right|^{2} & h_{2} h_{3}^{*}+h_{1} h_{2}^{*} \\
0 & -h_{2}^{*} h_{3}-\left|h_{1}\right|^{2} & \left|h_{1}\right|^{2}+\left|h_{3}\right|^{2} & -h_{1} h_{2}^{*}-\left|h_{3}\right|^{2} \\
0 & h_{2}^{*} h_{3}+h_{1}^{*} h_{2} & -h_{1}^{*} h_{2}-\left|h_{3}\right|^{2} & \left|h_{2}\right|^{2}+\left|h_{3}\right|^{2}
\end{array}\right]+\sigma^{2} \mathbf{I}_{4}
$$

The performance index of $\mathbf{R}_{1}$ is defined by $I_{4}\left(\mathbf{R}_{1}\right)=\frac{1}{2(M-1)}\left[\left(\sum_{j=1}^{M} \frac{\left|r_{1, j}\right|}{\left|r_{1,1}\right|}-1\right)+\left(\sum_{i=1}^{M} \frac{\left|r_{i, 1}\right|}{\left|r_{1,1}\right|}-1\right)\right]$, where $M=4$ denotes the order of matrix $\mathbf{R}_{1}, r_{i, j}$ denotes the element on the $i$ th row and the $j$ th column of matrix $\mathbf{R}_{1}$. The lower the $I_{4}\left(\mathbf{R}_{1}\right)$ value, the better the performance.

(ii) If $s_{i}$ belongs to modulation scheme II, then it can be easily obtained that

$$
\begin{aligned}
\mathbf{R}_{1} & =\lim _{N \rightarrow \infty}\left[\frac{1}{N / 4} \mathbf{Y}_{1} \mathbf{Y}_{1}{ }^{H}\right] \\
& =\left[\begin{array}{cccc}
\left(\left|h_{1}\right|^{2}+\left|h_{2}\right|^{2}+\left|h_{3}\right|^{2}\right) E\left(|s|^{2}\right)+\sigma^{2} & \left(h_{1} h_{2}^{*}-h_{1}^{*} h_{2}\right) E\left(s^{2}\right) & \left(-h_{1} h_{3}^{*}+h_{1}^{*} h_{2}\right) E\left(s^{2}\right) & \left(h_{1} h_{3}^{*}-\left|h_{2}\right|^{2}\right) E\left(s^{2}\right) \\
\left(-h_{1} h_{2}^{*}+h_{1}^{*} h_{2}\right) E\left(s^{* 2}\right) & \left(\left|h_{1}\right|^{2}+\left|h_{2}\right|^{2}\right) E\left(|s|^{2}\right)+\sigma^{2} & \left(-h_{2} h_{3}^{*}-\left|h_{1}\right|^{2}\right) E\left(|s|^{2}\right) & \left(h_{2} h_{3}^{*}+h_{1} h_{2}^{*}\right) E\left(|s|^{2}\right) \\
\left(-h_{1}^{*} h_{3}+h_{1} h_{2}^{*}\right) E\left(s^{* 2}\right) & \left(-h_{2}^{*} h_{3}-\left|h_{1}\right|^{2}\right) E\left(|s|^{2}\right) & \left(\left|h_{1}\right|^{2}+\left|h_{3}\right|^{2}\right) E\left(|s|^{2}\right)+\sigma^{2} & \left(-h_{1} h_{2}^{*}-\left|h_{3}\right|^{2}\right) E\left(|s|^{2}\right) \\
\left(h_{1}^{*} h_{3}-\left|h_{2}\right|^{2}\right) E\left(s^{* 2}\right) & \left(h_{2}^{*} h_{3}+h_{1}^{*} h_{2}\right) E\left(|s|^{2}\right) & \left(-h_{1}^{*} h_{2}-\left|h_{3}\right|^{2}\right) E\left(|s|^{2}\right) & \left(\left|h_{2}\right|^{2}+\left|h_{3}\right|^{2}\right) E\left(|s|^{2}\right)+\sigma^{2}
\end{array}\right]
\end{aligned}
$$

2. If the received samples are reconstructed as (24), then the reshaped samples can be expressed as:

$$
\mathbf{Y}_{3}=\left[\begin{array}{cccccc}
h_{1} & h_{2} & h_{3} & 0 & 0 & 0 \\
0 & 0 & 0 & h_{2} & -h_{1} & 0 \\
-h_{3}^{*} & 0 & h_{1}^{*} & 0 & 0 & 0 \\
0 & 0 & 0 & h_{3} & -h_{2} & 0
\end{array}\right]\left[\begin{array}{cccc}
s_{1} & s_{4} & \cdots & s_{3 N_{b}-2} \\
s_{2} & s_{5} & \cdots & s_{3 N_{b}-1} \\
s_{3} & s_{6} & \cdots & s_{3 N_{b}} \\
s_{1}^{*} & s_{4}^{*} & \cdots & s_{3 N_{b}-2}^{*} \\
s_{2}^{*} & s_{5}^{*} & \cdots & s_{3 N_{b}-1}^{*} \\
s_{3}^{*} & s_{6}^{*} & \cdots & s_{3 N_{b}}^{*}
\end{array}\right]+\left[\begin{array}{cccc}
w_{1} & w_{5} & \cdots & w_{N-3} \\
w_{2} & w_{6} & \cdots & w_{N-2} \\
w_{3}^{*} & w_{7}^{*} & \cdots & w_{N-1}^{*} \\
w_{4} & w_{8} & \cdots & w_{N}
\end{array}\right]
$$


(i) If $s_{i}$ belongs to modulation scheme I, then it can be easily obtained that

$$
\mathbf{R}_{3}=\lim _{N \rightarrow \infty}\left[\frac{1}{N / 4} \mathbf{Y}_{3} \mathbf{Y}_{3}^{H}\right]=\left[E\left(|s|^{2}\right)\right]\left[\begin{array}{cccc}
\left|h_{1}\right|^{2}+\left|h_{2}\right|^{2}+\left|h_{3}\right|^{2} & 0 & 0 & 0 \\
0 & \left|h_{1}\right|^{2}+\left|h_{2}\right|^{2} & 0 & h_{2} h_{3}^{*}+h_{1} h_{2}^{*} \\
0 & 0 & \left|h_{1}\right|^{2}+\left|h_{3}\right|^{2} & 0 \\
0 & h_{2}^{*} h_{3}+h_{1}^{*} h_{2} & 0 & \left|h_{2}\right|^{2}+\left|h_{3}\right|^{2}
\end{array}\right]+\sigma^{2} \mathbf{I}_{4}
$$

(ii) If $s_{i}$ belongs to modulation scheme II, then it can be easily obtained that

$$
\begin{aligned}
\mathbf{R}_{3} & =\lim _{N \rightarrow \infty}\left[\frac{1}{N / 4} \mathbf{Y}_{3} \mathbf{Y}_{3}{ }^{H}\right] \\
& =\left[\begin{array}{cccc}
\left(\left|h_{1}\right|^{2}+\left|h_{2}\right|^{2}+\left|h_{3}\right|^{2}\right) E\left(|s|^{2}\right)+\sigma^{2} & \left(h_{1} h_{2}^{*}-h_{1}^{*} h_{2}\right) E\left(s^{2}\right) & 0 & \left(h_{1} h_{3}^{*}-\left|h_{2}\right|^{2}\right) E\left(s^{2}\right) \\
\left(h_{1}^{*} h_{2}-h_{1} h_{2}^{*}\right) E\left(s^{* 2}\right) & \left(\left|h_{1}\right|^{2}+\left|h_{2}\right|^{2}\right) E\left(|s|^{2}\right)+\sigma^{2} & -h_{2} h_{3} E\left(s^{* 2}\right) & \left(h_{2} h_{3}^{*}+h_{1} h_{2}^{*}\right) E\left(|s|^{2}\right) \\
0 & -h_{2}^{*} h_{3}^{*} E\left(s^{2}\right) & \left(\left|h_{1}\right|^{2}+\left|h_{3}\right|^{2}\right) E\left(|s|^{2}\right)+\sigma^{2} & -h_{3}^{* 2} E\left(s^{2}\right) \\
\left(h_{3} h_{1}^{*}-\left|h_{2}\right|^{2}\right) E\left(s^{* 2}\right) & \left(h_{2}^{*} h_{3}+h_{1}^{*} h_{2}\right) E\left(|s|^{2}\right) & -h_{3}^{2} E\left(s^{* 2}\right) & \left(\left|h_{2}\right|^{2}+\left|h_{3}\right|^{2}\right) E\left(|s|^{2}\right)+\sigma^{2}
\end{array}\right]
\end{aligned}
$$

\subsection{Feature extraction of OSTBC3 with rate $1 / 2$ 's coded signal}

The received samples of OSTBC3 with rate 1/2's coded signal can be expressed as:

$$
\begin{aligned}
& \mathbf{y}=\left[\begin{array}{llll}
\mathbf{y}_{1} & \mathbf{y}_{2} & \cdots & \mathbf{y}_{N_{b}}
\end{array}\right]=\left[\begin{array}{llll}
y_{1} & y_{2} & \cdots & y_{N}
\end{array}\right] \\
& =\left[\begin{array}{l}
h_{1} \\
h_{2} \\
h_{3}
\end{array}\right]^{T}\left[\begin{array}{ccccccccc}
s_{1} & -s_{2} & -s_{3} & -s_{4} & s_{1}^{*} & -s_{2}^{*} & -s_{3}^{*} & -s_{4}^{*} & \cdots \\
s_{2} & s_{1} & s_{4} & -s_{3} & s_{2}^{*} & s_{1}^{*} & s_{4}^{*} & -s_{3}^{*} & \cdots \\
s_{3} & -s_{4} & s_{1} & s_{2} & s_{3}^{*} & -s_{4}^{*} & s_{1}^{*} & s_{2}^{*} & \cdots
\end{array}\right. \\
& \cdots s_{4 N_{b}-3}-s_{4 N_{b}-2}-s_{4 N_{b}-1}-s_{4 N_{b}} s_{4 N_{b}-3}^{*}-s_{4 N_{b}-2}^{*}-s_{4 N_{b}-1}^{*} \quad-s_{4 N_{b}}^{*} \\
& \cdots s_{4 N_{b}-2} s_{4 N_{b}-3} \quad s_{4 N_{b}} \quad-s_{4 N_{b}-1} s_{4 N_{b}-2}^{*} s_{4 N_{b}-3}^{*} \quad s_{4 N_{b}}^{*} \quad-s_{4 N_{b}-1}^{*}
\end{aligned}
$$

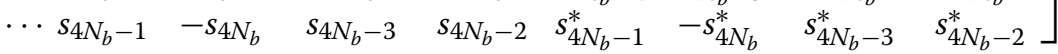

$$
\begin{aligned}
& +\left[\begin{array}{llll}
w_{1} & w_{2} & \cdots & w_{N}
\end{array}\right]
\end{aligned}
$$

If the received samples are reshaped as (8), then the reshaped samples can be expressed as:

$$
\mathbf{Y}_{1}=\left[\begin{array}{cccc}
h_{1} & h_{2} & h_{3} & 0 \\
h_{2} & -h_{1} & 0 & -h_{3} \\
h_{3} & 0 & -h_{1} & h_{2} \\
0 & h_{3} & -h_{2} & -h_{1}
\end{array}\right]\left[\begin{array}{ccccc}
s_{1} & s_{1}^{*} & \cdots & s_{4 N_{b}-3} & s_{4 N_{b}-3}^{*} \\
s_{2} & s_{2}^{*} & \cdots & s_{4 N_{b}-2} & s_{4 N_{b}-2}^{*} \\
s_{3} & s_{3}^{*} & \cdots & s_{4 N_{b}-1} & s_{4 N_{b}-1}^{*} \\
s_{4} & s_{4}^{*} & \cdots & s_{4 N_{b}} & s_{4 N_{b}}^{*}
\end{array}\right]+\left[\begin{array}{cccc}
w_{1} & w_{5} & \cdots & w_{N-3} \\
w_{2} & w_{6} & \cdots & w_{N-2} \\
w_{3} & w_{7} & \cdots & w_{N-1} \\
w_{4} & w_{8} & \cdots & w_{N}
\end{array}\right]
$$

It can be easily obtained that

$$
\begin{aligned}
& \mathbf{R}_{1}=\lim _{N \rightarrow \infty}\left[\frac{1}{N / 4} \mathbf{Y}_{1} \mathbf{Y}_{1}{ }^{H}\right] \\
& =E\left(|s|^{2}\right)\left[\begin{array}{cccc}
\left(\left|h_{1}\right|^{2}+\left|h_{2}\right|^{2}+\left|h_{3}\right|^{2}\right) & h_{1} h_{2}^{*}-h_{1}^{*} h_{2} & h_{1} h_{3}^{*}-h_{1}^{*} h_{3} & h_{2} h_{3}^{*}-h_{2}^{*} h_{3} \\
h_{1}^{*} h_{2}-h_{1} h_{2}^{*} & \left(\left|h_{1}\right|^{2}+\left|h_{2}\right|^{2}+\left|h_{3}\right|^{2}\right) & h_{2} h_{3}^{*}-h_{2}^{*} h_{3} & h_{1}^{*} h_{3}-h_{1} h_{3}^{*} \\
h_{1}^{*} h_{3}-h_{1} h_{3}^{*} & h_{2}^{*} h_{3}-h_{2} h_{3}^{*} & \left(\left|h_{1}\right|^{2}+\left|h_{2}\right|^{2}+\left|h_{3}\right|^{2}\right) & h_{1} h_{2}^{*}-h_{1}^{*} h_{2} \\
h_{2}^{*} h_{3}-h_{2} h_{3}^{*} & h_{1} h_{3}^{*}-h_{1}^{*} h_{3} & h_{1}^{*} h_{2}-h_{1} h_{2}^{*} & \left(\left|h_{1}\right|^{2}+\left|h_{2}\right|^{2}+\left|h_{3}\right|^{2}\right)
\end{array}\right]+\sigma^{2} \mathbf{I}_{4}
\end{aligned}
$$

Furthermore,

$$
\operatorname{Re}\left(\mathbf{R}_{1}\right)=\left[E\left(|s|^{2}\right)\left(\left|h_{1}\right|^{2}+\left|h_{2}\right|^{2}+\left|h_{3}\right|^{2}\right)+\sigma^{2}\right] \mathbf{I}_{4}
$$

The performance can be measured by $I_{1}\left[\operatorname{Re}\left(\mathbf{R}_{1}\right)\right]$. The lower the $I_{1}\left[\operatorname{Re}\left(\mathbf{R}_{1}\right)\right]$ value, the better the performance. 


\section{Classification of space-time code}

\subsection{Analysis of the features of different STBCs}

In this subsection, we analyze the features of different STBCs mentioned in Section 3. The features of different STBCs are summarized in Table 1. It should be noted that the features extracted from the reconstructed receiver are independent of the channel matrix. Figure 1 illustrates these features in the form of decision tree. First, SM and Alamouti codes are recognized by the values of Amari1 $=$ $10 \log \left[I_{1}\left(\mathbf{R}_{1}\right)\right]$, Amari2 $=10 \log \left[I_{2}\left(\mathbf{R}_{1}\right)\right]$, and Amari5 = $10 \log [I(\mathbf{T})]$. Then the OSTBC3 with rate $1 / 2$ is recognized by the values of Amari3 $=10 \log \left[I_{1} \operatorname{Re}\left(\mathbf{R}_{1}\right)\right]$. Finally, Amari4 $=10 \log \left[I_{4}\left(\mathbf{R}_{1}\right)\right]$ and Amari6 $=10 \log \left[I_{3}\left(\mathbf{R}_{3}\right)\right]$ are used to distinguish the first code of OSTBC3 with rate $3 / 4$ from the second code of OSTBC 3 with rate $3 / 4$. Unfortunately, it appears to be difficult to find theoretical values of these thresholds. Therefore, we propose SVM-based classifier for the blind recognition of five linear STBCs in the next subsection.

\subsection{Classification of space-time code by support vector machine}

SVM has demonstrated good performance in numerous pattern recognition problems [16-21]. It can discriminate non-linear separable data by mapping original feature space into a higher dimensional space via kernel functions. The commonly used kernel functions are as follows [16]:

$$
K(\mathbf{x}, \mathbf{y})=(\langle\mathbf{x}, \mathbf{y}\rangle+1)^{p}(\text { Polynomial })
$$

Table 1 The features of different STBCs

\begin{tabular}{ll}
\hline Code & Features \\
\hline SM & Amari1 $<\varepsilon_{1}$; Amari5 $>\varepsilon_{5}$ \\
Alamouti & If $s_{i}$ belongs to modulation scheme I, Amari1 $<\varepsilon_{1}$ \\
& and Amari5 $<\varepsilon_{5} ;$ \\
& If $s_{i}$ belongs to modulation scheme II, Amari1 $>\varepsilon_{1}$, \\
& Amari5 $<\varepsilon_{5}$, Amari2 $<\varepsilon_{2}$, \\
& Amari3 $<\varepsilon_{3}$ \\
$\frac{3}{4}$ OSTBC3 & Amari1 $>\varepsilon_{1}$, Amari2 $>\varepsilon_{2}$, Amari3 $>\varepsilon_{3} ;$ \\
Code 1 & If $s_{i}$ belongs to modulation scheme I, Amari4 $>\varepsilon_{4}$, \\
& Amari6 $<\varepsilon_{6}$ \\
& If $s_{i}$ belongs to modulation scheme II, Amari4 $>\varepsilon_{4}$, \\
& Amari6 $<\varepsilon_{6}$ \\
$\frac{3}{4}$ OSTBC3 & Amari1 $>\varepsilon_{1}$, Amari2 $>\varepsilon_{2}$, Amari3 $>\varepsilon_{3} ;$ \\
Code 2 & If $s_{i}$ belongs to modulation scheme I, Amari4 $<\varepsilon_{4}$, \\
& Amari6 $<\varepsilon_{6}$ \\
\hline$\frac{1}{2}$ OSTBC3 & If $s_{i}$ belongs to modulation scheme II, Amari4 $>\varepsilon_{4}$, \\
& Amari6 $>\varepsilon_{6}$
\end{tabular}

Amari1 $=10 \log \left[I_{1}\left(\mathbf{R}_{1}\right)\right]$, Amari2 $=10 \log \left[I_{2}\left(\mathbf{R}_{1}\right)\right]$, Amari3 $=10 \log \left[I_{1} \operatorname{Re}\left(\mathbf{R}_{1}\right)\right]$, Amari4 $=10 \log \left[I_{4}\left(\mathbf{R}_{1}\right)\right]$, Amari5 $=10 \log [/(\mathbf{T})]$, Amari6 $=10 \log \left[I_{3}\left(\mathbf{R}_{3}\right)\right] . \varepsilon_{1}, \varepsilon_{2}$, $\varepsilon_{3}, \varepsilon_{4}, \varepsilon_{5}$, and $\varepsilon_{6}$ are thresholds to discriminate the features of different STBCs.

$$
\begin{aligned}
& K(\mathbf{x}, \mathbf{y})=e^{-\|\mathbf{x}-\mathbf{y}\|^{2} / 2 \sigma^{2}}(\text { Gaussian }) \\
& K(\mathbf{x}, \mathbf{y})=\tanh (\kappa \mathbf{x} \cdot \mathbf{y}-\delta)(\text { Sigmoidal })
\end{aligned}
$$

In this paper, we select a polynomial function as the kernel function of SVM classifier. Figures 2 and 3 illustrate the feature distribution of five STBCs in two different three-dimensional spaces projected by the sixdimensional feature space of Amari1, Amari2, Amari3, Amari4, Amari5, and Amari6. The number of received samples, the type of modulation, the number of Monte Carlo trials, and the signal-to-noise ratio (SNR) are $N=$ 2048, QPSK, 50 times, and $20 \mathrm{~dB}$, respectively. It shows that SM and Alamouti cluster at different places in the three-dimensional spaces mapped by Amari1, Amari2, and Amari5; OSTBC3 with rate $1 / 2$, first code of OSTBC3 with rate $3 / 4$ and second code of OSTBC3 with rate $3 / 4$ cluster at different place in the three-dimensional spaces mapped by Amari3, Amari4, and Amari6. Therefore, five STBCs can be separated easily by a hyperplane in the sixdimensional feature space. The optimal hyperplane for classification is obtained from the training set based on the criteria of maximizing the margin and minimizing the empirical risk.

The proposed algorithm is composed of two parts: training process (Algorithm 1) and recognition process (Algorithm 2).

\section{Algorithm 1 Training process \\ Training process is achieved by training data and is com- posed of four steps:}

1: Reshape the training data as (8) and compute $\mathbf{R}_{1}$; compute the values of Amari1 $=10 \log \left[I_{1}\left(\mathbf{R}_{1}\right)\right]$, Amari2 $=10 \log \left[I_{2}\left(\mathbf{R}_{1}\right)\right]$, Amari3 = $10 \log \left[I_{1} \operatorname{Re}\left(\mathbf{R}_{1}\right)\right]$, and Amari4 = $10 \log \left[I_{4}\left(\mathbf{R}_{1}\right)\right]$.

2: Reconstruct the training data as (11) and compute $\mathbf{C}_{4}^{[1,2]}=c_{4}\left[\mathbf{Y}_{2}(:, i), \mathbf{Y}_{2}(:, i)^{H}, \mathbf{Y}_{2}(1, i), \mathbf{Y}_{2}(2, i)^{H}\right]$; compute matrix $\mathbf{T}=\mathbf{U}^{\dagger} \hat{\mathbf{H}}_{1}$ and the value of Amari5 $=10 \log [I(\mathbf{T})]$, where $\mathbf{U}$ is obtained from eigen-decompose of $\mathbf{C}_{4}^{[1,2]}$ and $\hat{\mathbf{H}}_{1}$ is obtained from $\mathbf{Y}_{2}$ by JADE.

3: Reconstruct the training data as (24) and compute $\mathbf{R}_{3}$; compute the value of Amari6 $=10 \log \left[I_{3}\left(\mathbf{R}_{3}\right)\right]$.

4: Map the values of Amari1, Amari2, Amari3, Amari4, Amari5, and Amari6 into a six-dimensional feature space via polynomial kernel functions of SVM and search the optimal hyperplane for classification.

It should be noted that the training data for the proposed classifier is different from the pilot data in the semi-blind channel estimation of communication system. 


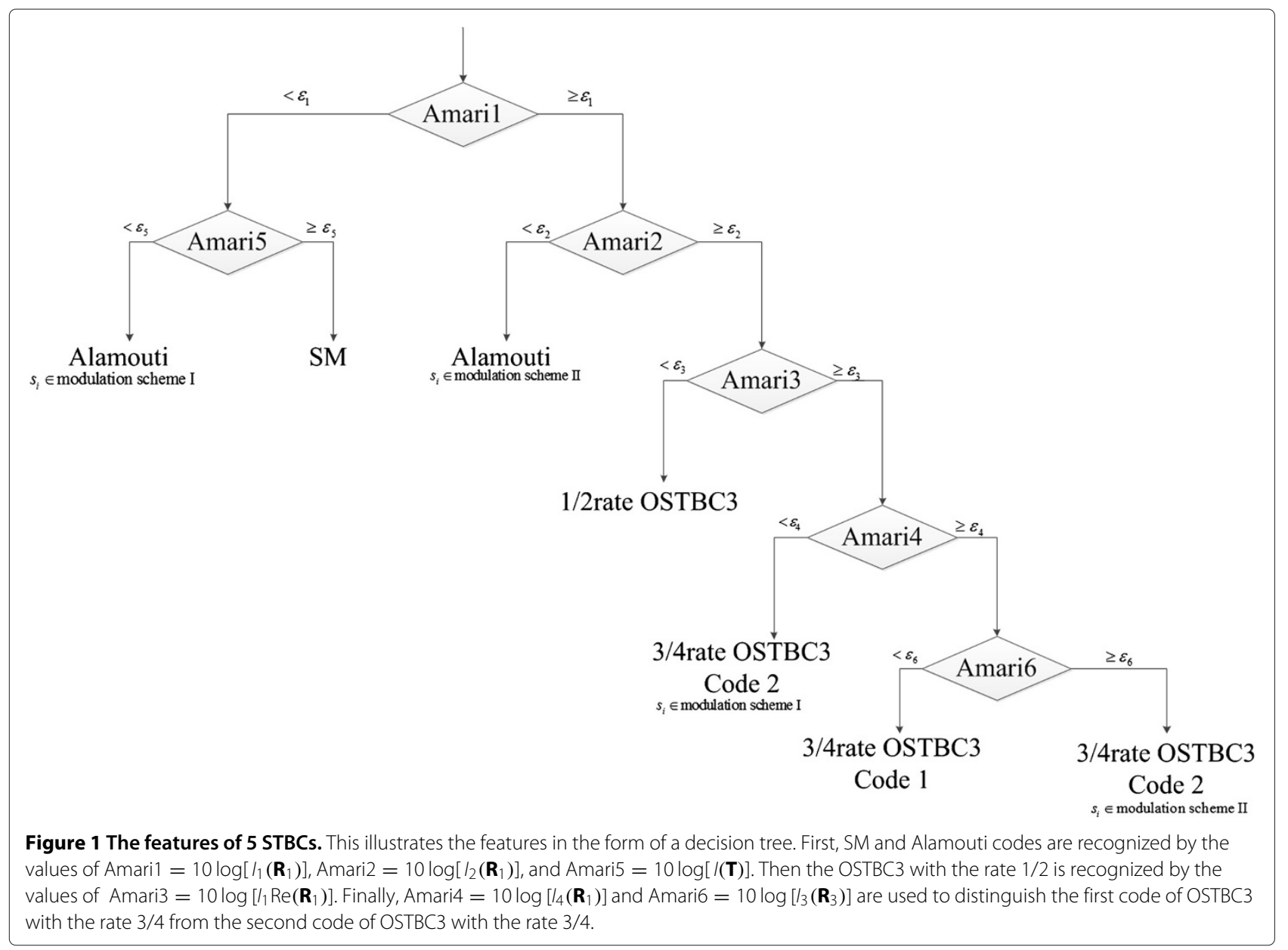

The optimal hyperplane for classification obtained by the training data is independent of CSI and source messages, and thus, it can be applied to all of the trials of recognition. However, the pilot data are indispensable in every trial of semi-blind channel estimation which incurs in a penalty in terms of bandwidth efficiency.

After training, a recognition process (Algorithm 2) is launched.

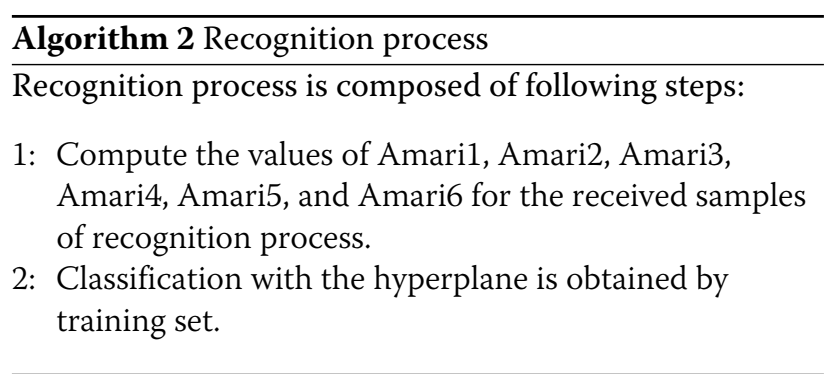

\section{Simulation results}

In this section, the performances of the proposed classifier are demonstrated by some simulation examples. First,
20 Monte Carlo trials of communications for each type of STBC in Section 2 are generated. For each communication, SNR varies from 0 to $20 \mathrm{~dB}$. The features extracted from these trials are employed as the training set of SVM. After training, a recognition process is launched. One thousand Monte Carlo trials are run for each type of STBC under the conditions that (1) a Rayleigh quasi-static fading channel, i.e., each element of channel, follows an i.i.d. circular Gaussian distribution with zero mean and unit variance, (2) there is a complex Gaussian circular and spatially uncorrelated noise, (3) there is only a single receive antenna in receiver side, and (4) SNR varies from $-10 \sim$ $20 \mathrm{~dB}$. SNR is defined as $\mathrm{SNR}=10 \log _{10}\left(P / \sigma^{2}\right)$ where $P=$ $(1 / l) E\left\{\operatorname{Tr}\left[\mathbf{C}(s) \mathbf{C}^{H}(s)\right]\right\}$ and $\sigma^{2}$ corresponds to the total power of transmitted signals and the power of additive white Gaussian noise, respectively. The channel matrix is randomly selected for each realization of training process and recognition process. The performance of proposed classifier is evaluated by the average probability of correct recognition which is defined as $p_{c}=\frac{N_{c}}{N_{\text {total }}}$, where $N_{\text {total }}=5,000$ is the total number of Monte Carlo trials and $N_{c}$ is the number of trials for which the space-time coding is correctly recognized. 


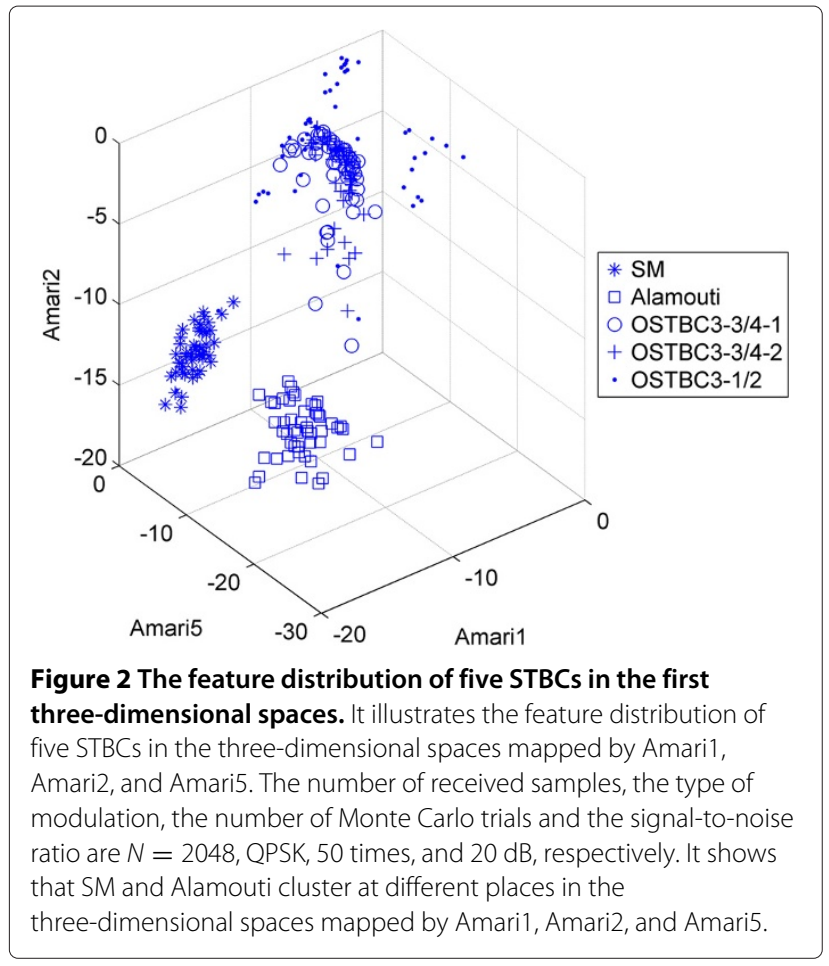

5.1 Influence of the number of received samples

In the first simulation, performances of the proposed classifier under different numbers of received samples $N$ are compared. Figure 4 shows the average probability of correct recognition for the proposed classifier in the case of

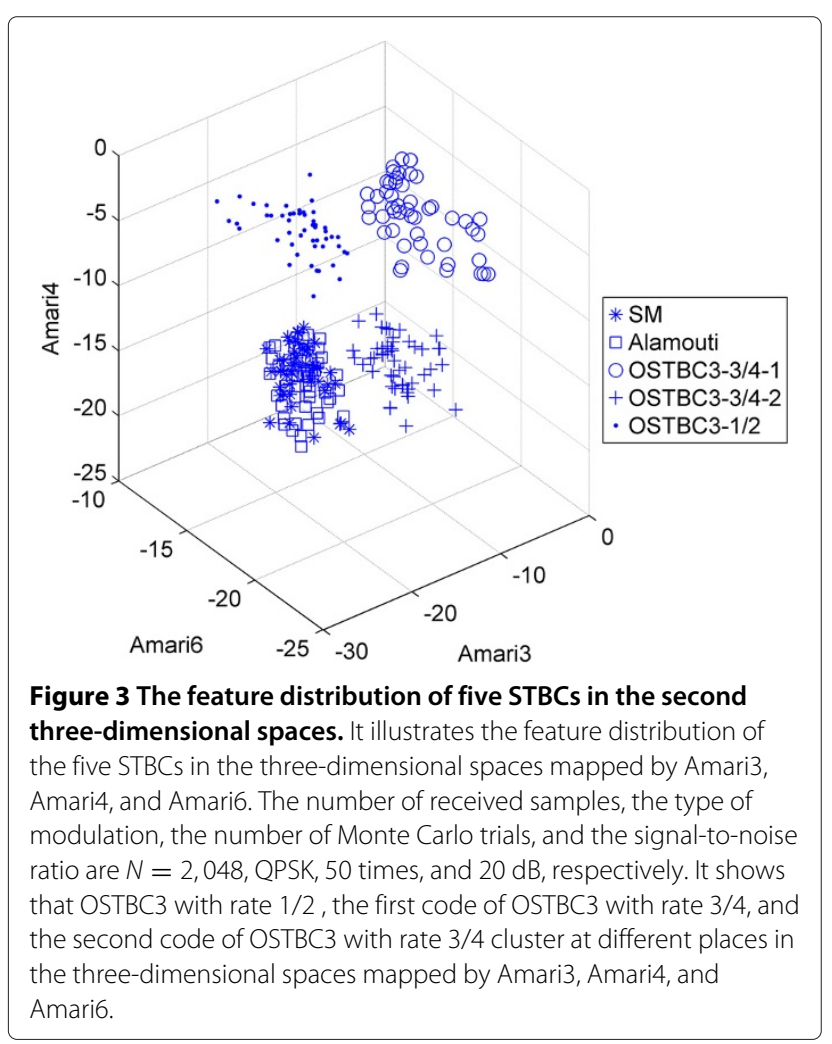

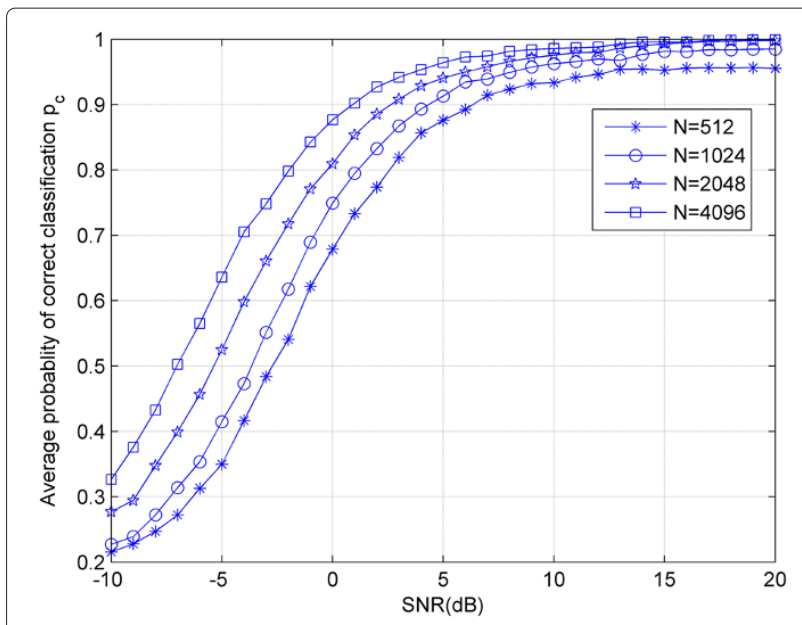

Figure 4 Influence of the number of received samples $\boldsymbol{N}$. It shows the average probability of correct recognition for the proposed classifier in the case of $N=512, N=1,024, N=2,048$, and $N=4,096$. It illustrates that the probability of correct recognition is improved by a greater number of received samples.

$N=512, N=1,024, N=2,048$, and $N=4,096$. It can be seen that the probability of correct recognition of the proposed classifier can reach up to 0.955 and 0.985 at high SNR when received samples are $N=512$ and $N=1,024$, respectively; the probability of correct recognition can reach 1 at high SNR when received samples are $N=2,048$ and $N=4,096$. The proposed SVM-based classifier's good performance of recognition is because it maps the features of five STBCs into a higher dimensional space which is much more identifiable than the one-dimensional space. Furthermore, Figure 4 illustrates that the probability of correct recognition is improved by

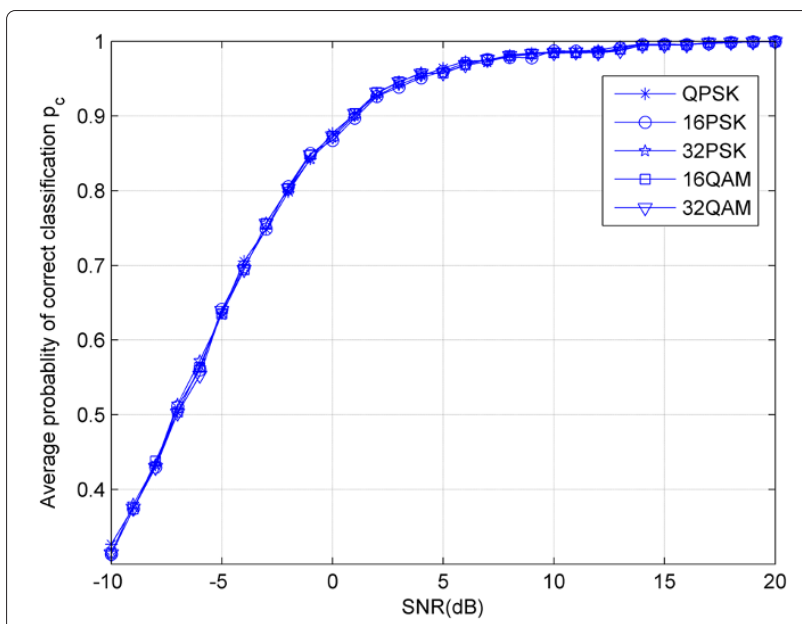

Figure $\mathbf{5}$ Influence of the modulation. It shows the average probability of correct recognition in the modulation of QPSK, 16PSK, 32PSK, 16QAM, and 32QAM. These modulations are mandatory for most of the wireless standards. It can be seen that our proposed classifier is robust to modulations. 


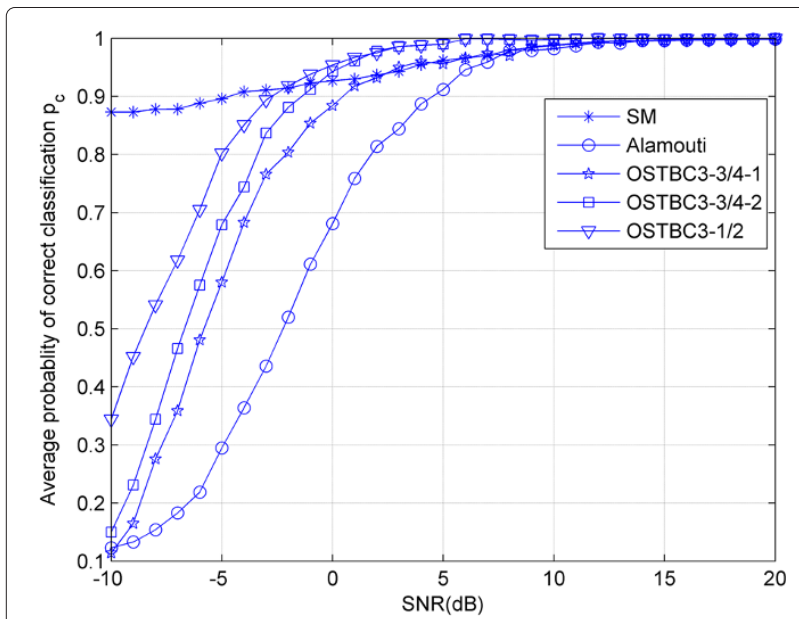

Figure 6 Influence of the space-time block code. It deals with the probability of correct recognition for each STBC. The number of received samples is equal to $N=4,096$ for each STBC, and the modulation adopted is QPSK. It shows that the proposed classifier has good performance of recognition for each STBC, and the probability of correct recognition can reach 1 at high SNR.

a greater number of received samples. The improvement is due to a better estimation of $\mathbf{R}_{1}, \mathbf{T}, \mathbf{R}_{3}$, and $\mathbf{R}_{4}$.

\subsection{Influence of the modulation}

In this simulation, performances of the proposed classifier in different modulations are compared to show the influence of the modulation. The number of received samples is equal to $N=4,096$. Figure 5 shows the average probability of correct recognition in the modulation of QPSK, 16PSK, 32PSK, 16QAM, and 32QAM. These modulations are mandatory for most of the wireless standards [10]. It can be seen from Figure 5 that our proposed classifier is robust to modulations.

\subsection{Influence of the space-time block code}

In this simulation, performances of the proposed algorithm for the correct recognitions of five STBCs are compared. The number of received samples is equal to $N=4,096$ for each STBC, and the modulation adopted is QPSK. Figure 6 deals with the average probability of correct recognition for each STBC. It shows that the proposed classifier has good performance of recognition for each STBC and the probability of correct recognition can reach 1 at high SNR. Furthermore, Figure 6 illustrates that proposed classifier seems to favor spatial multiplexing over other STBCs at low SNR. However, at high SNR, it first recognizes OSTBC3 with rate $1 / 2$, then it detects the second code of OSTBC3 with rate $3 / 4$, the first code of OSTBC3 with rate 3/4, spatial multiplexing, and Alamouti code.

\section{Conclusions}

In this paper, a novel algorithm is proposed to deal with the problem of blind recognition of linear STBCs in the MISO system. Utilizing the space-time redundancy, the original MISO model is transformed into a MIMO model by reconstructing the received samples. It is shown that the second- and higher-order statistics of the reconstructed receiver possess distinguishable features for different STBCs. Based on the high-dimensional feature space mapped by these features, an SVM-based classifier is trained to recognize the candidate STBCs without prior knowledge of CSI and modulation. Simulations show that the proposed method is capable of recognizing STBCs with high performance and robust to modulations. Future research will focus on the theoretical values of the thresholds for different features.

\section{Appendix 1}

Based on (12) and (13), $\mathbf{C}_{4}^{[1,2]}$ can be expressed as:

$$
\begin{aligned}
& \mathbf{C}_{4}^{[1,2]}=c_{4}\left[\mathbf{Y}_{2}(:, i), \mathbf{Y}_{2}(:, i)^{H}, \mathbf{Y}_{2}(1, i), \mathbf{Y}_{2}(2, i)^{H}\right]
\end{aligned}
$$

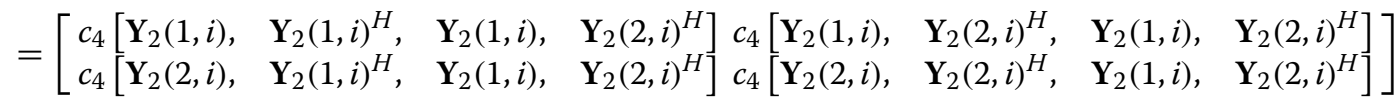

$$
\begin{aligned}
& =\mathbf{H} c_{4}\left(\mathbf{S}(:, i), \quad \mathbf{S}^{H}(:, i), \quad \mathbf{Y}_{2}(1, i), \quad \mathbf{Y}_{2}(2, i)^{H}\right) \mathbf{H}^{H}
\end{aligned}
$$

where

$$
\begin{aligned}
& c_{4}\left(\mathbf{S}(:, i), \quad \mathbf{S}^{H}(:, i), \quad \mathbf{Y}_{2}(1, i), \quad \mathbf{Y}_{2}(2, i)^{H}\right)
\end{aligned}
$$

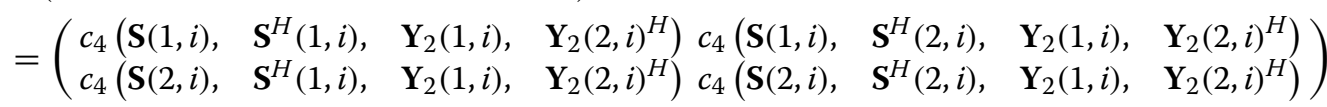

Using the properties of the cumulants and the independence of $s_{i}$, it can be easily obtained that

$$
\begin{aligned}
& c_{4}\left(\mathbf{S}(1, i), \quad \mathbf{S}^{*}(1, i), \quad \mathbf{Y}_{2}(1, i), \quad \mathbf{Y}_{2}(2, i)^{H}\right) \\
& =c_{4}\left(\mathbf{S}(1, i), \quad \mathbf{S}^{*}(1, i), \quad h_{1} \mathbf{S}(1, i)+\cdots h_{n_{t}} \mathbf{S}\left(n_{t}, i\right), \quad h_{1} \mathbf{S}\left(n_{t}+1, i\right)+\cdots h_{n_{t}} \mathbf{S}\left(2 n_{t}, i\right)\right) \\
& =0
\end{aligned}
$$


Similarly,

$$
\begin{array}{llll}
c_{4}(\mathbf{S}(1, i), & \mathbf{S}^{*}(2, i), & \mathbf{Y}_{2}(1, i), & \left.\mathbf{Y}_{2}(2, i)^{H}\right)=0 ; \\
c_{4}(\mathbf{S}(2, i), & \mathbf{S}^{*}(1, i), & \mathbf{Y}_{2}(1, i), & \left.\mathbf{Y}_{2}(2, i)^{H}\right)=0 ; \\
c_{4}(\mathbf{S}(2, i), & \mathbf{S}^{*}(2, i), & \mathbf{Y}_{2}(1, i), & \left.\mathbf{Y}_{2}(2, i)^{H}\right)=0 .
\end{array}
$$

\section{Appendix 2}

Similar to the proof of Appendix 1, $\mathbf{C}_{4}^{[1,2]}$ can be expressed as:

$$
\begin{aligned}
& \mathbf{C}_{4}^{[1,2]}=c_{4}\left[\mathbf{Y}_{2}(:, i), \quad \mathbf{Y}_{2}(:, i)^{H}, \quad \mathbf{Y}_{2}(1, i), \quad \mathbf{Y}_{2}(2, i)^{H}\right]
\end{aligned}
$$

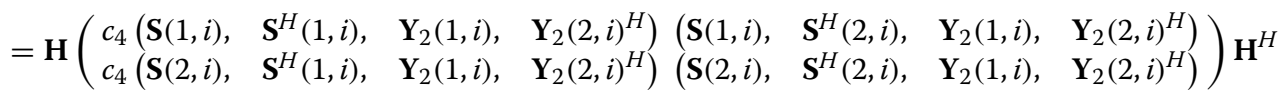

Using the properties of the cumulants and the independence of $s_{i}$, it can be easily obtained that

$$
\begin{aligned}
& c_{4}\left(\mathbf{S}(1, i), \quad \mathbf{S}^{*}(1, i), \quad \mathbf{Y}_{2}(1, i), \quad \mathbf{Y}_{2}(2, i)^{H}\right) \\
& =c_{4}\left(\mathbf{S}(1, i), \quad \mathbf{S}^{*}(1, i), \quad h_{1} \mathbf{S}(1, i)+h_{2} \mathbf{S}(2, i), \quad h_{2} \mathbf{S}^{*}(1, i)-h_{1} \mathbf{S}^{*}(2, i)\right) \\
& =k_{4} h_{1} h_{2}
\end{aligned}
$$

Similarly,

$$
\begin{aligned}
& c_{4}\left(\mathbf{S}(1, i), \quad \mathbf{S}^{*}(2, i), \quad \mathbf{Y}_{2}(1, i), \quad \mathbf{Y}_{2}(2, i)^{H}\right)=0 ; \\
& c_{4}\left(\mathbf{S}(2, i), \quad \mathbf{S}^{*}(1, i), \quad \mathbf{Y}_{2}(1, i), \quad \mathbf{Y}_{2}(2, i)^{H}\right)=0 ; \\
& c_{4}\left(\mathbf{S}(2, i), \quad \mathbf{S}^{*}(2, i), \quad \mathbf{Y}_{2}(1, i), \quad \mathbf{Y}_{2}(2, i)^{H}\right)=-k_{4} h_{1} h_{2} .
\end{aligned}
$$

\section{Abbreviations}

CSI: Channel state information; JADE: Joint approximate diagonalization of eigenmatrices; MIMO: Multiple-input multiple-output; MISO: Multiple-input single-output; ML: Maximum likelihood; OSTBC3: Orthogonal STBC using three transmit antennas; SM: Spatial multiplexing; STBCs: Space-time block codes; SVM: Support vector machine.

\section{Competing interests}

The authors declare that they have no competing interests.

\section{Acknowledgments}

The work and the contribution were partially supported by Science and Technology on Electronic Information Control Laboratory and partially supported by Science and Technology on Communication Information Security Control Laboratory under grant 9140C130304120C13064.

Received: 25 March 2013 Accepted: 30 May 2013

Published: 13 June 2013

\section{References}

1. E Larsson, P Stoica, Space-Time Block Coding for Wireless Communications. (Cambridge University Press, Cambridge, 2003)

2. S Alamouti, A simple transmit diversity technique for wireless communications. IEEE J. Sel. Areas Commun. 16, 1451-1458 (1998)

3. H Jafarkhani, A quasi-orthogonal space-time block code. IEEE Trans.Commun. 49, 1-4 (2001)

4. AG S Shahbazpanahi, J Manton, Closed-form blind MIMO channel estimation for orthogonal space-time block codes. IEEE Trans. Signal Process. 53, 4506-4517 (2005)

5. N Ammar, Z Ding, Channel identifiability under orthogonal space-time coded modulations without training. IEEE Trans. Wireless Commun. $\mathbf{5}$ 1003-1013 (2006)

6. N Ammar, Z Ding, Blind channel identifiability for generic linear space-time block codes. IEEE Trans. Signal Process. 55, 202-217 (2007)

7. J Via, I Santamaria, On the blind identifiability of orthogonal space time block codes from second order statistics. IEEE Trans. Inf. Theory. 54, 709-722 (2008)
8. V Choqueuse, GBLC, Yao Mansour A K, Blind channel estimation for STBC systems using higher-order statistics. IEEE Trans. Wireless Commun. 10, 495-505 (2011)

9. YBN M Shi, W SU. STC and BLAST MIMO modulation recognition. Proceedings of IEEE GLOBLECOM (IEEE Piscataway, 2007), pp. 3034-3039

10. V Choqueuse, LC, K Yao, G Burel, Hierarchical space-time block code recognition using correlation matrices. IEEE Trans. Wireless Commun. 7, 3526-3534 (2008)

11. V Choqueuse, LC, K Yao, G Burel. Blind recognition of linear space time block codes. Proceedings of IEEE Conference Acoustic, Speech and Signal Processing, Las Vegas, NV, 2008 (IEEE Piscataway, 2008), pp. 2833-2836

12. V Choqueuse, LCKY, M Marazin, G Burel, Blind recognition of linear space time block codes: a likelihood-based approach. IEEE Trans. Signal Process. 58, 1290-1299 (2010)

13. MG Luo, L LGan, Blind recognition of space-time block code using correlation matrices in a high dimensional feature space. J. Inf. Comput. Sci. 9, 1469-1476 (2012)

14. AC S Amari, HH Yang, ed. by MCM D S Touretzky, ME Hasselmo. A new learning algorithm for blind signal separation. Neural Information Processing Systems, (1996), pp. 757-763

15. J Cardoso, A Souloumiac, Blind beamforming for non-Gaussian signals. IEE Proc. F Radar Signal Process. 140, 16-27 (1993)

16. C Burges, A tutorial on support vector machines for pattern recognition. Data Min. Knowl. Discov. 2, 121-167 (1998)

17. J Weston, C Watkins, in Proceeding of the Seventh European Artificial Neural Networks, ed. by Y Smith. Support vector machines for multi-class pattern recognition (Bruges, 1999), pp. 219-224

18. RS EL Allwein, Y Singer, Reducing multiclass to binary: a unifying approach for margin classifiers. Mach. Learn. Res. 1, 113-141 (2001)

19. C Hsu, C Lin, A comparison of methods for multiclass support vector machines. IEEE Trans. Neural Netw. 13, 415-425 (2002)

20. A Smola, BS Ikopf, A tutorial on support vector regression. Stat. Comput. 14, 199-222 (2004)

21. JH A Ganapathiraju, J Picone, Applications of support vector machines to speech recognition. IEEE Trans. Signal Process. 52, 2348-2355 (2004)

doi:10.1186/1687-1499-2013-164

Cite this article as: Qian et al:: Blind recognition of space-time block code in MISO system. EURASIP Journal on Wireless Communications and Networking 2013 2013:164 